\title{
J-Shaped Upper Mini-Sternotomy Versus Full Sternotomy for Aortic Valve Replacement: A Comparative Study
}

\author{
Álvaro Borrero, MD, ${ }^{1}$ Tatiana Samboni, ${ }^{2}$ Natalia Prado, MD,${ }^{1}$ Diana Cristina Carrillo-Gómez, MD, $M S c,{ }^{3}$ \\ German Camilo Giraldo-González, MD ${ }^{2,3}$ Liliana Flórez-Elvira, MSc, ${ }^{4}$ Eduardo Cadavid-Alvear, $\mathrm{MD}^{1}$ \\ ${ }^{1}$ Cardiovascular Surgery Unit, Fundación Valle del Lili, Colombia; ${ }^{2}$ Health Sciences Faculty, Icesi University, Colombia; ${ }^{3}$ Cardiology \\ Service, Heart Failure Unit, Fundación Valle del Lili, Colombia; ${ }^{4}$ Clinical Research Center, Fundación Valle del Lili, Colombia
}

\section{ABSTRACT}

Background: This study aims to compare the characteristics between patients who underwent aortic valve replacement (AVR) through a J-shaped upper mini-sternotomy (UMS) and patients who underwent full sternotomy (FS) in the basis of clinical care and hospital outcomes.

Methods: A retrospective, cross-sectional study was conducted on adult patients who were subjected to AVR by UMS from 2014 to 2017, compared with a historical control of patients who had undergone UMS by FS from 2011 to 2014. Patients, who received combined valve replacement or aortic surgery, as well as heart valve reinterventions due to endocarditis, were excluded. Sociodemographic characteristics, medical history, hospital and intensive care stay, blood transfusions, complications, and mortality of both procedures were compared.

Results: There were 57 patients under UMS and 99 patients under FS included in this study. The median age was 67 years, and $56.77 \%$ of the patients were male. No differences were observed in the past medical history and the type of valve implanted between the groups. During surgery, patients under UMS received a lower percentage of red blood cell and platelet transfusions compared with FS. However, UMS had a higher percentage of cryoprecipitate transfusion. Intensive care stay was shorter in UMS compared with FS (three days; interquartile range $[\mathrm{IQR}], 2-4$; and four days; IQR, 2-6, respectively) without differences in overall hospital stay, postoperative complications, in-hospital mortality, and 30-day mortality.

Conclusions: The J-shaped upper mini-sternotomy is a feasible surgical technique that does not increase inhospital or 30-day mortality, neither hospital stay nor infectious complications.

\section{INTRODUCTION}

Aortic valve disease, aortic stenosis (AS) aortic or regurgitation (AR), is associated with cardiovascular complications,

Received December 1, 2019; received in revised form March 2, 2020; accepted March 13, 2020.

Correspondence: Carrillo-Gómez Diana, Av Simón Bolivar, Cra 98 \# 18-49 4th Floor, 1st Tower, Cardiology Service, Fundación Valle del Lili, Cali, Colombia, 760032; 57-2-3319090,_Ext. 7453 (e-mail: diana_cristinac@hotmail.com). including death. Therefore, aortic valve replacement (AVR) has been the definitive option for most patients [Glauber 2015; Salenger 2016]. Traditionally, the surgical approach in patients with aortic valvulopathy is full sternotomy (FS) with cardiopulmonary bypass [Mill 2017]. Over the last 20 years, there has been an interest in reducing complications, trauma, and risks associated with surgical interventions through minimally invasive approaches, including J-shaped upper mini sternotomy (UMS), reversed-C mini-sternotomy, L ministernotomy, $\mathrm{T}$ mini-sternotomy and mini-thoracotomies, which involve smaller incisions than FS for faster postoperative recovery, without increasing mortality and with lesser blood transfusion requirements [Glauber 2015; Kaczmarczyk 2015; Johnston 2015; Svensson 2007; Cohn 1997].

Real-life studies have shown equivalent results of ministernotomy to FS, without increasing mortality [Glauber 2015], suggesting a shorter hospital stay, faster recovery and lesser use of health resources [Gilmanov 2013]. In our population, the results of this technique in patients with AVR are unknown. The intra- and postoperative characteristics of patients undergoing AVR by UMS are described below and

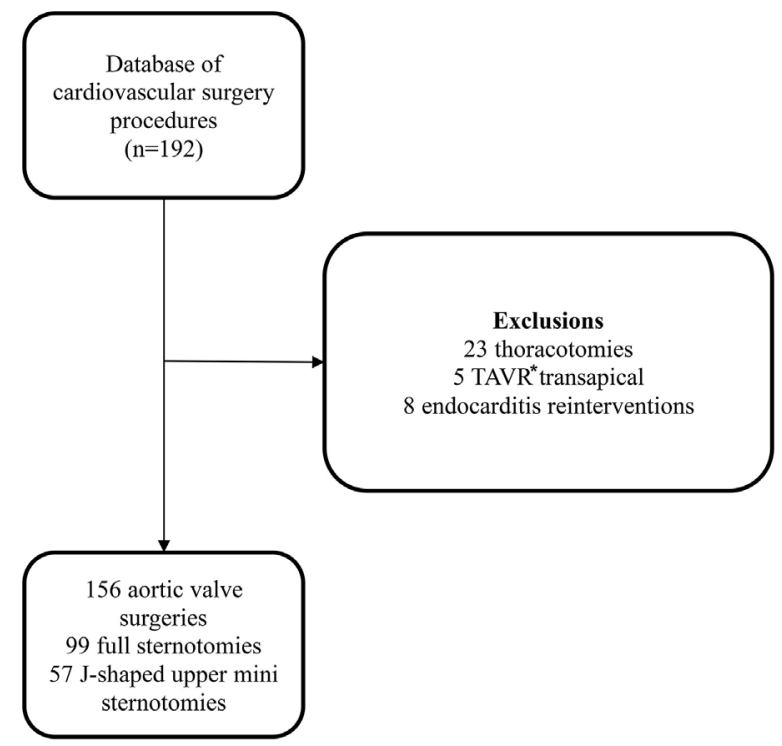

*TAVR: Transcatheter Aortic-Valve Replacement

Figure 1. Flowchart of the study. 


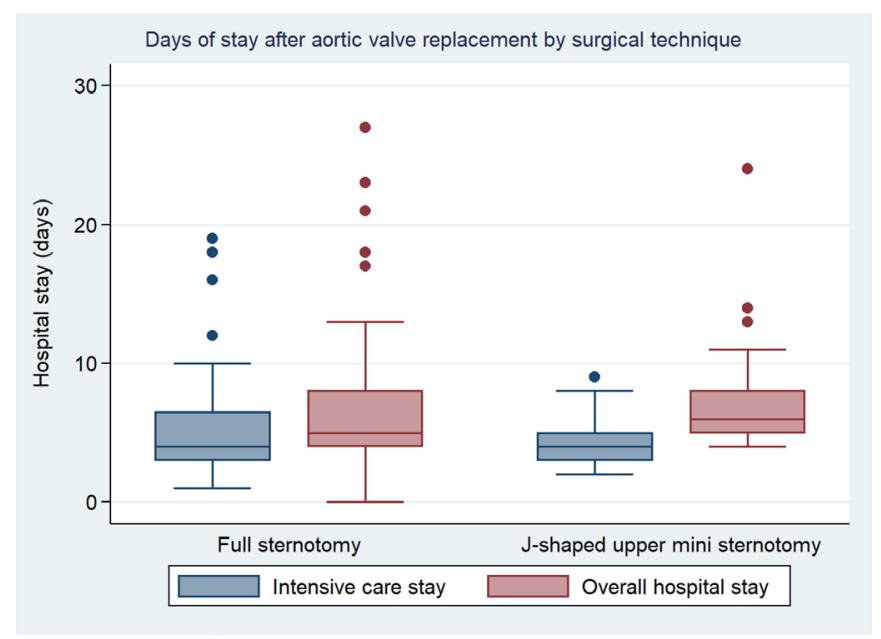

Figure 2. Box plot of the hospital and intensive care stay after aortic valve replacement.

are compared with historical control patients undergoing AVR by FS before the implementation of the UMS in terms of hospital stay, mortality, and use of blood components.

\section{MATERIALS AND METHODS}

A retrospective, cross-sectional study was conducted on patients undergoing AVR by UMS, a procedure that was implemented in 2014 at the Fundación Valle del Lili in Cali, Colombia. Patients over 18 years of age undergoing AVR, with stenosis or aortic regurgitation, were eligible. Patients were compared with a historical control of individuals, who had undergone AVR by FS between 2011 to 2014. To this date, all the unique aortic valve replacement procedures are carried out by UMS, in accordance with the institutional protocol. Individuals with combined valve surgery, AVR with intervention in the ascending aorta or with concomitant revascularization or re-interventions due to endocarditis were excluded from the study, since these procedures, in the period from 2014 to 2017, would have gone to FS.

Sociodemographic data, echocardiographic findings, preoperative laboratory information, type of procedure, aortic clamp and perfusion time, intra- and postoperative transfusion of blood products, complications, and mortality data were obtained from the patients' medical records. Hospital stay, days of invasive mechanical ventilation, and hemodynamic support required in the immediate postoperative period also were included. Intensive care days were defined as the number of days from surgery until the time of transfer to general hospitalization rooms. Hospital stay included the days from surgery until hospital discharge. The study was approved by the institutional biomedical research ethics committee.

Univariate statistical analysis of sociodemographic characteristics, medical history and pre- and postoperative findings was performed. These findings were illustrated in $2 \mathrm{xn}$ tables. Quantitative variables were described as mean or

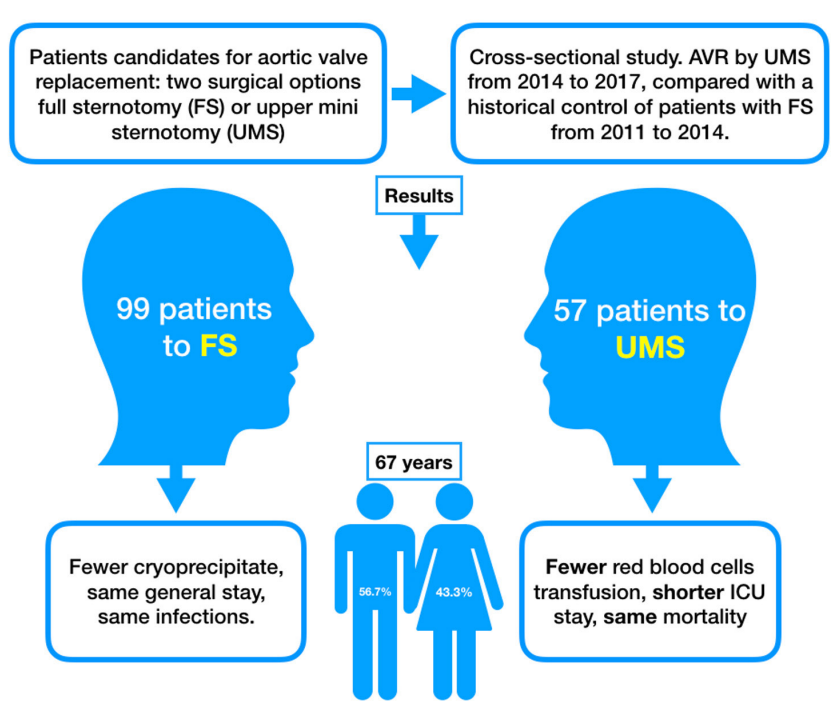

Implications
The J-shaped upper mini sternotomy is a feasible surgical technique, does not
increase in-hospital or 30 days mortality, neither hospital stay or infectious
complications, with fewer red blood cells transfusion and ICU stay

Figure 3. Graphical abstract

median using dispersion measures, such as standard deviation $(\mathrm{SD})$ or interquartile range (IQR). Qualitative variables were expressed as absolute numbers and percentages. Outcomes of interest were intensive care stay, hospital stay, transfusions, and in-hospital mortality. A Wilcoxon-Mann-Whitney range test was used to compare the hospital stay, and a Fisher's exact test was used to examine the percentage of blood transfusions and in-hospital deaths.

\section{RESULTS}

There were 192 patients eligible for this study from the cardiovascular surgery database (Figure 1). Among these patients, 155 were included, $63.22 \%$ were FS and $36.77 \%$ were UMS. The median age was 67 years (IQR, 58-74) without differences between the groups, and $56.77 \%$ were male. Arterial hypertension followed by diabetes mellitus were the relevant past medical history more frequently observed. Sixty-five percent of individuals had impairment of functional class and less often angina as symptoms related to heart valve disease. No differences were observed in the end-systolic or end-diastolic diameters of the left ventricle between the two groups, and the median left ventricular ejection fraction (LVEF) was normal (Table 2). A reduced LVEF was observed in about $15.46 \%$ and $12.28 \%$ of patients in the FS group and UMS, respectively $(P=.38)$.

The main indication for surgery in $85.82 \%$ of patients was aortic stenosis (AS), followed by aortic regurgitation (AR) in $12.91 \%$. The remaining cases sought surgery of combined AS and AR and aortic papillary fibroelastoma. No differences were shown in pre-surgical laboratory tests 
Table 1. Sociodemographic characteristics of individuals with aortic valve replacement by surgical technique

\begin{tabular}{|c|c|c|c|}
\hline Variable & $\begin{array}{l}\text { Full sternotomy } \\
\qquad(N=99)\end{array}$ & UMS $(N=57)$ & $P$ \\
\hline Age (years) & $67(58-74)$ & $67(57-74)$ & .73 \\
\hline \multicolumn{4}{|l|}{ Gender } \\
\hline Female & $46(46.46)$ & $23(40.35)$ & .28 \\
\hline Male & $53(53.54)$ & $34(59.65)$ & \\
\hline Coronary heart disease & $13(13.83)$ & $2(4.26)$ & .07 \\
\hline \multicolumn{4}{|l|}{ NYHA } \\
\hline I & $14(16.47)$ & $24(43.64)$ & .01 \\
\hline II & $45(52.94)$ & $16(29.09)$ & \\
\hline III & $20(23.53)$ & $10(18.18)$ & \\
\hline IV & $6(7.06)$ & $5(9.09)$ & \\
\hline Angina & $14(16.47)$ & $24(43.64)$ & \\
\hline Diabetes mellitus & $15(15.31)$ & $9(15.79)$ & 1.00 \\
\hline Arterial hypertension & $60(61.86)$ & $29(50.88)$ & .23 \\
\hline \multicolumn{4}{|l|}{ Smoking } \\
\hline Never & $66(80.49)$ & $43(75.44)$ & .45 \\
\hline Actual & $5(6.10)$ & $2(3.51)$ & \\
\hline Former & $11(13.41)$ & $12(21.05)$ & \\
\hline Extracardiac arteriopathy & $3(3.37)$ & 0 & .28 \\
\hline Stroke & $3(3.03)$ & $1(1.75)$ & 1.00 \\
\hline COPD & $6(6.12)$ & $2(3.51)$ & .71 \\
\hline Atrial fibrillation & $6(6.06)$ & $1(1.75)$ & .42 \\
\hline Myocardial infarction & $4(4.17)$ & $2(3.51)$ & 1.00 \\
\hline $\mathrm{PCl}$ & $3(3.09)$ & $2(3.51)$ & 1.00 \\
\hline CKD & $6(6.12)$ & $1(1.75)$ & .26 \\
\hline Use of anticoagulants & $6(6.06)$ & $3(5.26)$ & 1.00 \\
\hline
\end{tabular}

CKD: Chronic kidney disease; COPD: Chronic obstructive lung disease; NYHA: New York Heart Association functional class; PCl: Percutaneous coronary intervention; UMS: J-shaped Upper Mini Sternotomy.

or the kind of implanted prosthesis between the two surgical techniques. The surgery status, surgical times and type of implanted prosthesis are illustrated in Table 3. A longer aortic clamp time was recorded in the UMS group concerning FS, with no differences in perfusion times between the two techniques.

Transfusions: Intraoperatively, a lower percentage of patients required red blood cell transfusions and platelet apheresis, and a higher percentage of patients required cryoprecipitate in USM (Table 3). Not so for fresh frozen plasma, in which there was no difference between the two techniques. In the postoperative period, there were no differences in the number of units of red blood cells, plasma, cryoprecipitate, or platelets apheresis transfused between the groups. The
Table 2. Preoperative echocardiographic findings

\begin{tabular}{|c|c|c|c|}
\hline Variable & Full sternotomy $(\mathrm{N}=99)$ & UMS $(N=57)$ & $P$ \\
\hline \multicolumn{4}{|l|}{ Echocardiography } \\
\hline LVEF (\%) & $63(55-70)$ & $62(58-70)$ & .71 \\
\hline $\operatorname{EDLVD}(\mathrm{mm})$ & $47(42-52)$ & $47(41-53)$ & .67 \\
\hline $\operatorname{ESLVD}(\mathrm{mm})$ & $29.5(26-36)$ & $30(24-38)$ & .76 \\
\hline Aortic root (mm) & $33(30-36)$ & $35(31-38.5)$ & .06 \\
\hline Ascending aorta $(\mathrm{mm})$ & $38(34-41)$ & $38(35-40)$ & .97 \\
\hline \multicolumn{4}{|l|}{ Aortic stenosis } \\
\hline None & $15(15.15)$ & $9(15.79)$ & .69 \\
\hline Mild & $1(1.01)$ & 0 & \\
\hline Moderate & $6(6.06)$ & $1(1.75)$ & \\
\hline Severe & 77 (77.78) & $47(82.46)$ & \\
\hline Aortic regurgitation & & & .96 \\
\hline None & $38(38.78)$ & $21(36.84)$ & \\
\hline Mild & $35(36.71)$ & $23(40.35)$ & \\
\hline Moderate & $9(9.18)$ & $5(8.77)$ & \\
\hline Severe & $16(16.33)$ & $8(14.04)$ & \\
\hline Tricuspid regurgitation & & & .45 \\
\hline None & $46(47.42)$ & $24(42.11)$ & \\
\hline Mild & $39(41.24)$ & $30(52.63)$ & \\
\hline Moderate & $9(9.28)$ & $3(5.26)$ & \\
\hline Severe & $2(2.06)$ & 0 & \\
\hline \multicolumn{4}{|l|}{ Mitral stenosis } \\
\hline Mild & $1(1.04)$ & $1(1.75)$ & 1.00 \\
\hline Mitral regurgitation & & & .75 \\
\hline None & $55(56.70)$ & $30(52.63)$ & \\
\hline Mild & $32(32.99)$ & $21(36.84)$ & \\
\hline Moderate & $9(9.28)$ & $6(10.53)$ & \\
\hline Endocarditis & $3(3.09)$ & $1(1.75)$ & 1.00 \\
\hline
\end{tabular}

EDLVD, End-diastolic left ventricular diameter; ESLVD, End-systolic left ventricular diameter; LVEF, Left ventricular ejection fraction; UMS, (J-shaped) Upper mini sternotomy

overall percentage of postoperative transfusions was $8.33 \%$ for FS and $14.29 \%$ for UMS $(P=.280)$.

Hospital stay and mortality: In the UMS group, there were fewer days of hospital stay in the intensive care and intermediate care units (Figure 2). The median intensive and intermediate care stay was three days (IQR, 2-4) in the UMS group and four days (IQR, 2-6) for FS $(P<.01)$, with no differences in the overall hospital stay (5 days IQR 4-7 and 5 days IQR 4-8 respectively, $P=.68)$. There were no differences in the need for definitive postoperative pacemaker implantation $(3.51 \%$ in UMS and $5.10 \%$ in FS, $P=1.00$ ), surgical site infection $(1.75 \%$ and $1.02 \%$, respectively, $P=.09)$, reintervention due 
Table 3. Blood analysis and intraoperative and postoperative findings

\begin{tabular}{|c|c|c|c|}
\hline Variable & $\begin{array}{l}\text { Full sternotomy } \\
\qquad(N=99)\end{array}$ & UMS $(N=57)$ & $P$ \\
\hline \multicolumn{4}{|l|}{ Pre-surgical blood analysis } \\
\hline Hemoglobin (g/dL) & $13.43(1.69)$ & $13.87(1.94)$ & .06 \\
\hline Hematocrit (\%) & $40.27(4.92)$ & $40.98(5.19)$ & .18 \\
\hline Platelets (cells/mm3) & $227(201-271)$ & $213(169-159)$ & .11 \\
\hline Leukocytes (cells/mm3) & $7.30(5.95-8.60)$ & $7.44(6.34-8.45)$ & .81 \\
\hline Creatinine $(\mathrm{mg} / \mathrm{dL})$ & $0.92(0.79-1.1)$ & $0.89(0.77-1)$ & .45 \\
\hline \multicolumn{4}{|l|}{ Surgical variables } \\
\hline \multicolumn{4}{|l|}{ Surgery state } \\
\hline Elective & $68(69.70)$ & $35(61.40)$ & .06 \\
\hline Urgent & $25(25.25)$ & $22(38.60)$ & \\
\hline Emergent & $5(5.05)$ & 0 & \\
\hline \multicolumn{4}{|l|}{ Prosthesis implanted } \\
\hline Bioprosthesis & $71(72.72)$ & $44(78.57)$ & .44 \\
\hline Mechanical prosthesis & $27(28.28)$ & $12(21.43)$ & \\
\hline Prosthesis number & $21(21-23)$ & $21(19-23)$ & .15 \\
\hline \multicolumn{4}{|l|}{ Operative times } \\
\hline Perfusion & $74(65-85)$ & $76(69-88)$ & .31 \\
\hline Aortic clamp & $57(50-68)$ & $62(56-72)$ & .02 \\
\hline \multicolumn{4}{|c|}{ Intraoperative blood transfusions (\%) } \\
\hline Red blood cells & $50(50.51)$ & $20(35.09)$ & .04 \\
\hline Fresh frozen plasma & $20(20.20)$ & $8(14.04)$ & .22 \\
\hline Cryoprecipitate & $7(7.14)$ & $10(17.54)$ & .04 \\
\hline Platelets apheresis & $25(25.25)$ & $3(5.26)$ & $<.01$ \\
\hline Postoperative open chest & $3(3.19)$ & 0 & .29 \\
\hline \multicolumn{4}{|l|}{ Postoperative care } \\
\hline $\begin{array}{l}\text { Extubation in the first } 12 \\
\text { hours }\end{array}$ & $81(81.82)$ & $53(92.98)$ & .04 \\
\hline \multicolumn{4}{|l|}{ Postoperative blood analysis } \\
\hline Hemoglobin $(\mathrm{g} / \mathrm{dL})$ & $11.1(10.0-11.8)$ & $10.9(10.2-11.7)$ & .79 \\
\hline Hematocrit (\%) & $32.7(30.3-35.3)$ & $32.3(30.2-34.2)$ & .42 \\
\hline \multicolumn{4}{|c|}{ Postoperative blood transfusions (\%) } \\
\hline Red blood cells & $8(8.25)$ & $8(14.04)$ & .19 \\
\hline Fresh frozen plasma & $2(2.06)$ & 0 & .39 \\
\hline Cryoprecipitate & $2(2.06)$ & 0 & .39 \\
\hline Platelet apheresis & $1(1.02)$ & 0 & .63 \\
\hline
\end{tabular}

UMS, (J-shaped) upper mini-sternotomy

to infection (0 and $3.09 \%$, respectively, $P=.24$ ), or hospital or 30-day death (UMS, $1.75 \%$, versus $\mathrm{FS}, 2.06 \%, P=.69$; 30 -day death, $1.79 \%$ in UMS and $0 \%$ in FS, $P=.37$ ). Only one patient $(1.75 \%)$ needed conversion from UMS to FS.

\section{DISCUSSION}

Minimally invasive surgical procedure, UMS, for AVR is safe to the traditional technique; it reduces intensive care stay and the need for intraoperative blood products without increasing hospital mortality compared with FS [Korach 2010]. At our institution, the condition that favors this type of technique as a single procedure is the AVR, and that is not a re-intervention.

Our results showed no differences in mortality, but expressed shorter intensive care stays without differences in the overall hospital stay. In a study by Johnston et al, who evaluated the UMS in AVR, low mortality (0.7\%) was observed without differences between UMS and FS. However, the sample size of their study was much larger than ours, which may explain the differences observed in mortality [Johnston 2015]. Lehmann et al, reported a mortality of $2.2 \%$ at 30 days with UMS similar to our results [Lehmann 2015]. With regard to hospital stay, shorter hospital stays in UMS was reported by Johnston et al, a finding not seen in our study. Additionally, a higher number of early extubations in the UMS group was not observed, as we did in the UMS group with respect to FS. In the Mini-Stern Trial study comparing UMS (118 patients) to FS (104 patients), no differences in hospital stay were reported between the two groups (9.5 days in UMS versus 8.6 days in FS) or in mortality $(3.08 \%$ at 6 weeks in UMS versus $1.04 \%$ in FS) [Nair 2018]. A shorter hospital stay was noted in our results compared to that of the Mini-Stern Trial (5 days for UMS and FC), especially in the intensive care unit stay in the UMS group, without differences in the overall stay.

The use of blood products was lower in UMS. Johnston et al, in the experience of the Cleveland Clinic, reported a lower overall use of blood products in the UMS group, tantamount to our results [Johnston 2015]. Lehmann et al, reported an average use of $2.1 \pm 4.2$ red blood cell packages, $0.2 \pm 1$. 1 platelet concentrate and $1.0 \pm 3.9$ of fresh frozen plasma transfusions in the UMS group, which was higher than ours, possibly due to the difference in the number of patients [Lehmann 2015].

Ghanta et al evaluated the database of the "Society of Thoracic Surgeons' to compare partial sternotomy or right thoracotomy (both called mini-AVR) with FS [Ghanta 2015]. In this study, differences in mortality, renal failure or stroke between the three techniques was not observed [Ghanta 2015]. A higher percentage of patients were discharged in the first four postoperative days, along with the consequent cost reduction in the mini-AVR group. There also was less time for invasive mechanical ventilation and blood transfusion [Ghanta 2015]. Ahangar et al, reported fewer infections, lesser pain, and shorter hospital stay in 30 patients with AVR using a right anterolateral technique compared with FS [Ahangar 2013]. In Germany, Shehada et al, in a retrospective analysis, found a similar 30-day mortality and, in patients with a minimal access AVR, significantly lower blood transfusions and shorter intubation time, being consistent with the lower blood requirements as in our study [Shehada 2016]. In six centers in Europe, Dalen $M$ et al, found similar mortality and survival up to 30 days and three years and, in the general 
cohort, a shorter bypass time in the mini-sternotomy group, which is a distinct finding from our study, and less packed red blood cell transfusions [Dalen 2016].

Our study has several limitations. First is the use of a historical control, which can introduce biases related to the changes over time in postoperative care strategies, such as the use of more restrictive transfusion protocols, earlier extubation and the greater experience of the surgical group, which may reduce the number of complications seen in the UMS group compared with FS. However, the surgical group in the two periods of time was the same as well as the postoperative care, although it cannot be affirmed which results have not been related to temporal variations. Second was the sample size of the UMS, which could underestimate the differences between the two groups at risk of type 1 error. However, our findings still are similar to the results previously reported. There were no differences in complications, despite the acquisition of the curve at the beginning of the implementation of the technique, a situation in favor of the safety of the procedure, and there was only one conversion to FS in the UMS group. Third was the non-evaluation of the pain scale to compare whether there is less or equal postoperative pain between the two techniques. Moreover, our findings do not apply to populations that are not individual AVRs. Patients in the UMS group were more frequently in New York Heart Association (NYHA) I (43.64\%) compared with FS (16.47\%); however, about $70 \%$ in both groups were in NYHA I or II functional class, and we believe that the varying symptoms are due to the most frequent angina reported in the UMS. We cannot assume if this is related to a different severity of the disease or different manifestations of the same disease.

\section{CONCLUSIONS}

In patients with aortic valve replacement needing as a single procedure, the use of the J-shaped upper mini-sternotomy technique may be safe, as it does not increase mortality and it reduces days of intensive care stay and the percentage of intraoperative transfusions with respect to full sternotomy.

\section{REFERENCES}

Ahangar AG, Charag AH, Wani ML, et al. 2013. Comparing aortic valve replacement through right anterolateral thoracotomy with median sternotomy. Int. Cardiovasc. Res. J. 7:90-4.

Cohn LH, Adams DH, Couper GS, et al. 1997. Minimally invasive cardiac valve surgery improves patient satisfaction while reducing costs of cardiac valve replacement and repair. Ann Surg 226:421-426.

Dalen M, Biancari F, Rubino AS, et al. 2016. Aortic valve replacement through full sternotomy with a stented bioprosthesis versus minimally invasive sternotomy with a sutureless bioprosthesis. Eur J Cardiothorac Surg 49:220-7.

Ghanta RK, Lapar DJ, Kern JA, et al. 2015. Minimally invasive aortic valve replacement provides equivalent outcomes at reduced cost compared with conventional aortic valve replacement: A real-world multiinstitutional analysis. J Thorac Cardiovasc Surg 149:1060-65.

Gilmanov D, Bevilacqua S, Murzi M, et al. 2013. Minimally invasive and conventional aortic valve replacement: a propensity score analysis. Ann Thorac Surg 96:837-43.

Glauber M, Ferrarini M, Miceli A. 2015. Minimally invasive aortic valve surgery: state of the art and future directions. Ann Cardiothorac Surg 4:26-32.

Johnston DR, Roselli EE. 2015. Minimally invasive aortic valve surgery: Cleveland Clinic experience. Ann. Cardiothorac. Surg.4:140-7.

Kaczmarczyk M, Szałański P, Zembala M, et al. 2015. Minimally invasive aortic valve replacement-pros and cons of keyhole aortic surgery. Kardiochirurgia i torakochirurgia polska $=$ Polish journal of cardio-thoracic surgery. 12:103-10.

Korach A, Shemin RJ, Hunter CT, Bao Y, Shapira OM. 2010. Minimally invasive versus conventional aortic valve replacement: a 10-year experience. J Cardiovasc Surg 51:417-21.

Lehmann S, Merk DR, Etz CD, et al. 2015. Minimally invasive aortic valve replacement: the Leipzig experience. Ann Cardiothorac Surg 4:49-56.

Mill MR, Anderson RH, Cohn LH. 2017. Surgical anatomy of the heart. In: Hill M, ed. Cardiac Surgery in the Adult. 5th ed. 21-42.

Nair SK, Sudarshan CD, Thorpe BS, et al. 2018. Mini-Stern Trial: A randomized trial comparing mini-sternotomy to full median sternotomy for aortic valve replacement. J Thorac Cardiovasc Surg 156:2124-2132. e2131.

Salenger R, Gammie JS, Collins JA. 2016. Minimally invasive aortic valve replacement. J Card Surg 31:38-50.

Shehada SE, Ozturk O, Wottke M, Lange R. 2016. Propensity score analysis of outcomes following minimal access versus conventional aortic valve replacement. Eur J Cardiothorac Surg 49: 464-469; 469-470.

Svensson LG. 2007. Minimally invasive surgery with a partial sternotomy "J" approach. Semin Thorac Cardiovasc Surg 19:299-303. 